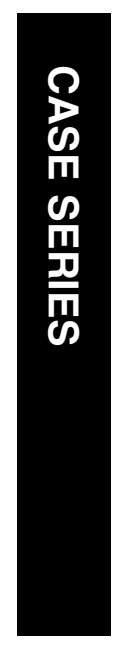

'Department of Ophthalmology, University Hospital Wales, Cardiff, UK

${ }^{2}$ Department of Ophthalmology, Sultan Qaboos University Hospital, Sultanate of Oman, Oman

${ }^{3}$ Department of Child Health, University Hospital Wales, Cardiff, UK

${ }^{4}$ Institute of Medical Genetics, University Hospital Wales, Cardiff, UK

${ }^{5}$ Department of Ophthalmology and Vision Sciences, The Hospital for Sick Children, Toronto, Ontario, Canada

${ }^{6}$ Department of Genetics, Mount Sinai Hospital, Toronto, Ontario, Canada

Correspondence: P Watts, Department of

Ophthalmology, University Hospital of Wales, Heath Park,

Cardiff CF 14 4XW, UK

Tel: +442920748583 ;

Fax: +44 2920748240

E-mail: Patrick.Watts@

cardiffandvale.wales.nhs.uk

Received: 10 September 2007

Accepted in revised form:

5 November 2007

Published online: 14

December 2007

\section{Chorioretinal dysplasia, hydranencephaly, and intracranial calcifications: pseudo-TORCH or a new syndrome?}

\author{
Abstract \\ Purpose To report the association of severe \\ chorioretinal dysplasia, hydranencephaly, \\ microcephaly, and intracranial calcification in \\ children with no evidence of intrauterine \\ infections. \\ Methods Two unrelated female infants with \\ visually inattentive behaviour, \\ hydranencephaly, and intracranial calcification \\ were referred for an ophthalmological \\ opinion. \\ Results The fundus examination and \\ computerised tomograms (CT scans) of head \\ were similar in both children. There was \\ bilateral extensive chorioretinal dysplasia, \\ intracranial calcifications, and \\ hydranencephaly. Serology was negative for \\ acquired intrauterine congenital infections. \\ Conclusions We report two cases that may \\ represent a new syndrome or the more severe \\ end of the spectrum of the pseudo-TORCH \\ (toxoplasma, rubella, cytomegalovirus, and \\ herpes simplex) syndrome. The association of \\ chorioretinal dysplasia with the pseudo- \\ TORCH syndrome has not been reported \\ previously. \\ Eye (2008) 22, 730-733; doi:10.1038/sj.eye.6703058; \\ published online 14 December 2007 \\ Keywords: chorioretinal dysplasia; \\ hydranencephaly; CNS calcifications
}

\section{Introduction}

The pseudo-TORCH syndrome, an autosomal recessive condition, is characterised by central nervous system findings similar to those seen in
P Watts ${ }^{1}$, N Kumar ${ }^{1}$, A Ganesh², P Sastry ${ }^{3}$, D Pilz ${ }^{4}$, AV Levin ${ }^{5}$ and D Chitayat ${ }^{6}$ intrauterine infections, but with negative serological tests for the typical TORCH infections (toxoplasmosis, rubella, cytomegalovirus, herpes, syphilis). ${ }^{1}$ Ophthalmologic abnormalities have not been consistently recognised in patients with the pseudo-TORCH syndrome. ${ }^{1,2}$

This report describes the association of hydranencephaly, intracranial calcifications, and chorioretinal dysplasia, which to our knowledge has not been reported previously.

\section{Methods}

Case 1

A 7-week-old female infant with roving eye movements was referred for ophthalmic evaluation. The mother had weakly positive immunoglobulin G (IgG) titres for CMV and herpes, and negative titres for rubella, toxoplasmosis, and syphilis. An antenatal computed tomography (CT) scan demonstrated hydranencephaly at 29 weeks.

The child had delayed developmental milestones, hypertonic reflexes, and a bilateral sensorineural hearing impairment. At 6 weeks, her head circumference had increased to $41 \mathrm{~cm}$, which required a ventriculoperitoneal shunt to prevent the hydranencephaly from expanding.

Ophthalmic examination revealed no visual responses to light in both eyes. Fundus examination revealed some vitreous haze with bilateral optic nerve hypoplasia, attenuated retinal vasculature, diffuse chorioretinal atrophy, pigment clumping, and a dysplastic retina (Figure 1a and $b$ ). A clinical diagnosis of a possible intrauterine infection was considered in view of the fundus findings. 
a

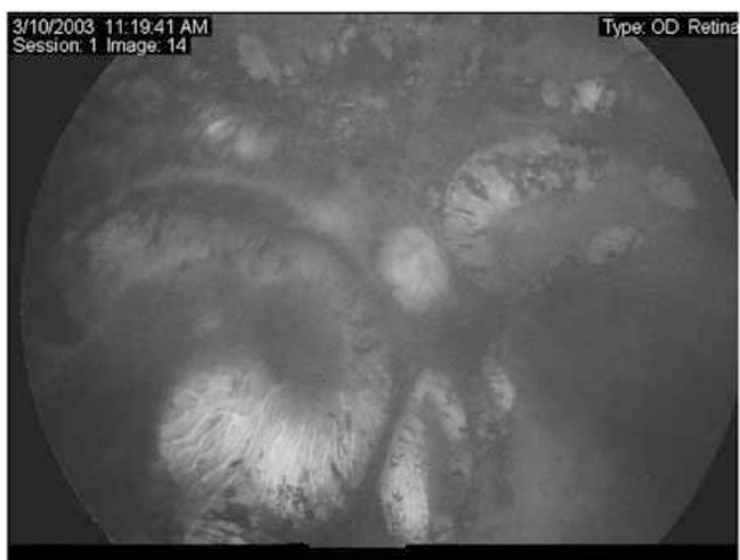

b

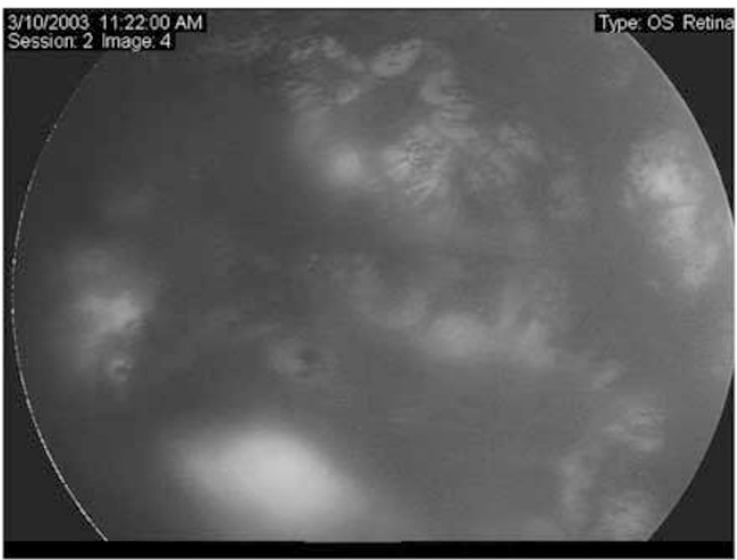

C

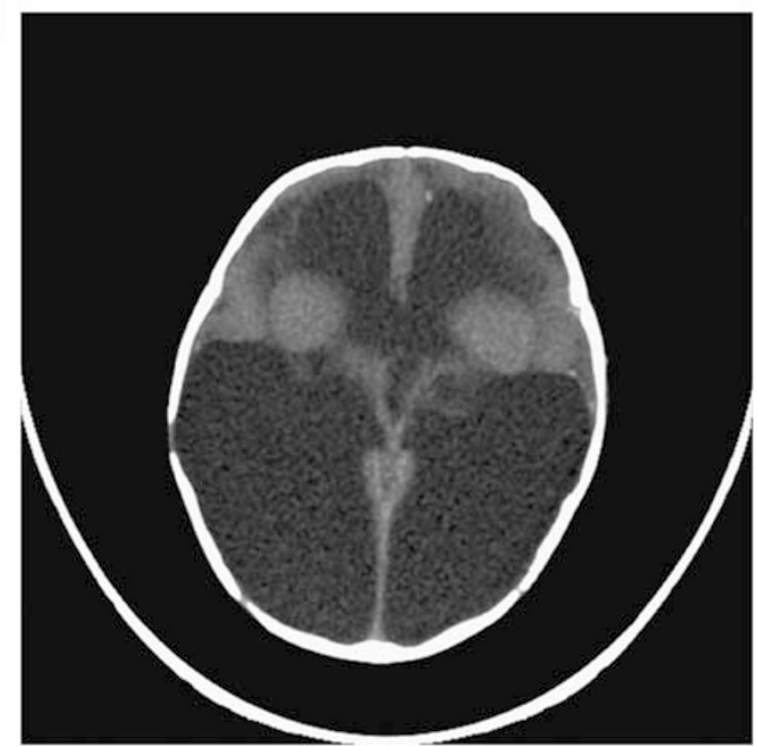

Figure 1 (a and b) Case 1. Vitreous haze with chorioretinal dysplasia with vessel attenuation in right and left eyes. (c) CT scan showing hydranencephaly with intracranial calcifications.

The creatine kinase (CK) level was 54 IU (normal 0-190 IU). Computerised scanning of the brain demonstrated hydranencephaly, an absent corpus callosum, and periventricular intracranial a

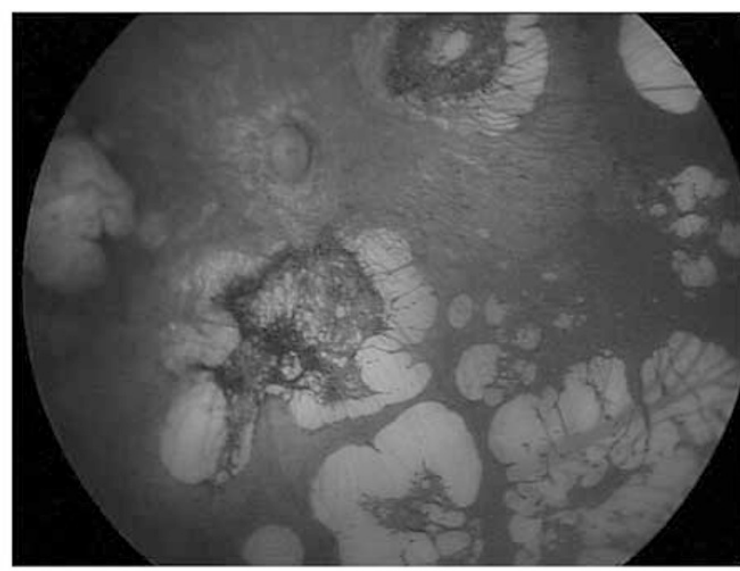

b

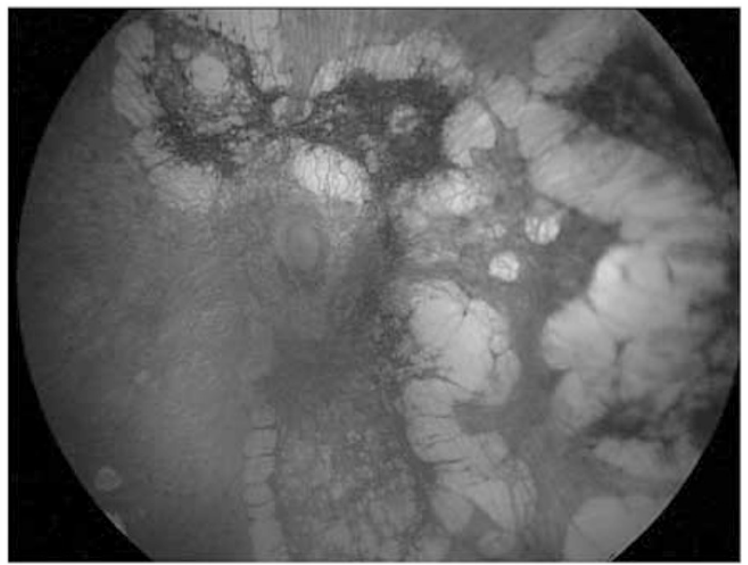

c

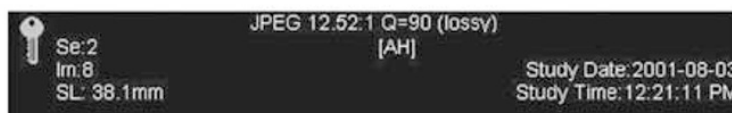

[R]

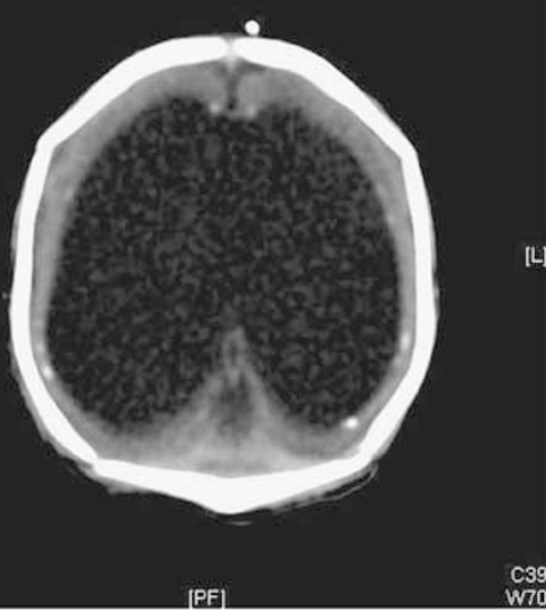

Figure 2 ( $a$ and $b$ ) Case 2. Chorioretinal dysplasia with vessel attenuation in right and left eyes. (c) CT scan showing hydranencephaly with intracranial calcifications.

calcifications (Figure 1c). Urine for CMV and serum TORCH screens were both negative. Chromosomal analysis revealed a normal female karyotype. 


\section{Case 2}

An unrelated 8-day-old female infant was referred for ophthalmic evaluation. She was the first child of healthy, unrelated parents, and was born at term by spontaneous, uncomplicated vaginal delivery. Her medical history was significant for the presence of hydranencephaly diagnosed antenatally and bilateral talipes equinovarus. Ocular examination revealed poor fixation following responses to light. Fundus examination showed bilateral optic nerve and macular hypoplasia, pigment clumping, extensive chorioretinal atrophy, and retinal dysplasia (Figure 2a and b). Cycloplegic refraction revealed a moderate myopia $(-4.0 \mathrm{D})$ in both eyes.

CK level was mildly elevated at $222 \mathrm{IU}$ (normal 0-190 IU). CT of the brain demonstrated microcephaly, hydranencephaly, lissencephaly, lobar holoprosencephaly, and multiple, coarse, periventricular calcifications (Figure 2c). TORCH serology did not suggest an intrauterine infection, and chromosomal analysis revealed a normal karyotype.

\section{Discussion}

Hydranencephaly, a congenital anomaly, has been hypothesised to occur owing to vascular insufficiency related to malformations or intrauterine infections. Optic nerve aplasia, absent retinal vessels, pigment epithelial abnormalities, and chorioretinitis have been reported with hydranencephaly. ${ }^{3-5}$ Extensive chorioretinal dysplasia, as seen in our cases, has not been described with hydranencephaly.

The diagnostic significance of intracranial calcification may be indicated by its location, intrauterine infections commonly associated with periventricular calcification and basal ganglia calcification with mitochondrial disease, although these sites of calcification may be seen in a number of other conditions and are not pathognomonic of a particular disease process. The location of calcification in our cases was periventricular, as has been described in intrauterine infections and in the pseudo-TORCH syndrome.

Anterior segment anomalies and retinal dysplasia have been described with muscle-eye-brain disease, WalkerWarburg syndrome, and Fukiyama congenital muscular dystrophy where congenital muscular dystrophy and lissencephaly features are prominent. In case 2, lissencephaly was associated with hydranencephaly and intracranial calcifications. The triad of chorioretinal lacunae, complex brain abnormalities including corpus callosum agenesis, and infantile spasms in female children constitute the diagnosis of Aicardi syndrome. ${ }^{6}$ The diffuse chorioretinal changes with hydranencephaly and the absence of infantile spasms make this diagnosis unlikely.

The pseudo-TORCH syndrome is characterised by microcephaly, spasticity, seizures, developmental delay, and intracranial calcification and negative laboratory workup for acquired intrauterine infections. ${ }^{2}$ However, none of the patients reported with this condition have been described to have fundus abnormalities.

Microphthalmia and cataracts have been reported with pseudo-TORCH syndrome ${ }^{5}$ (Table 1).

In summary, the cases presented have some features similar to the MEB syndrome, WWS, FCMD, Aicardi syndrome, and the pseudo-TORCH syndrome. However, the characteristic diffuse chorioretinal dysplasia with poor vision, associated with extensive hydranencephaly,

Table 1 Differential diagnosis

\begin{tabular}{|c|c|c|c|c|c|}
\hline Differential diagnosis & $\begin{array}{c}\text { Pseudo-TORCH } \\
\text { syndrome }\end{array}$ & $\begin{array}{c}\text { Aicardi } \\
\text { syndrome }\end{array}$ & $\begin{array}{c}\text { Walker-Warburg } \\
\text { syndrome }\end{array}$ & $\begin{array}{c}\text { Muscle eye brain } \\
\text { disease }\end{array}$ & $\begin{array}{c}\text { Congenital TORCH } \\
\text { infections }\end{array}$ \\
\hline \multicolumn{6}{|l|}{ Clinical features } \\
\hline Inheritance & AR & $\mathrm{XL}$ & AR & AR & - \\
\hline Epilepsy & + & + & + & + & + \\
\hline Chorioretinal dysplasia & + & - & + & + & - \\
\hline Chorioretinal lacunae & - & + & - & - & - \\
\hline Previous choroiditis & - & - & - & - & + \\
\hline \multicolumn{6}{|l|}{ Neuro-radiology } \\
\hline Periventricular calcification & + & - & $+/-$ & - & + \\
\hline Hydranencephaly & + & - & + & + & + \\
\hline \multicolumn{6}{|l|}{ Investigations } \\
\hline Elevated creatine kinase & - & - & + & + & - \\
\hline Urine CMV & - & - & - & - & + \\
\hline
\end{tabular}

$\mathrm{AR}=$ autosomal recessive; $\mathrm{CMV}=$ cytomegalovirus; $\mathrm{TORCH}=$ toxoplasma, rubella, cytomegalovirus, herpes simplex; $\mathrm{XL}=\mathrm{X}$ linked. 
intracranial calcifications, and developmental delay, makes these two cases distinctive. As far as we are aware, this is the first series to report an association of hydranencephaly, intracranial calcifications, and chorioretinal dysplasia and may represent part of a continuing spectrum of pseudo-TORCH or a new syndrome.

\section{References}

1 Vivarelli R, Grosso S, Cioni M, Galluzzi P, Monti L, Morgese $\mathrm{G}$ et al. Pseudo-TORCH syndrome or
Baraitser-Reardon syndrome: diagnostic criteria. Brain Dev 2001; 23(1): 18-23.

2 Knoblauch H, Tennstedt C, Brueck W, Hammer H, Vulliamy $\mathrm{T}$, Dokal I et al. Two brothers with findings resembling congenital intrauterine infection-like syndrome (pseudoTORCH syndrome). Am J Med Genet A 2003; 120(2): 261-265.

3 Hill K, Gogan DG, Dodge PR. Ocular signs associated with hydranencephaly. Am J Ophthalmol 1961; 51: 267-275.

4 Manschot WA. Eye findings in hydranencephaly. Ophthalmologica 1971; 162(3): 151-159.

5 Storm RL, PeBenito R. Bilateral optic nerve aplasia associated with hydranencephaly. Ann Ophthalmol 1984; 16(10): 988-992.

6 Aicardi J. Aicardi syndrome. Brain Dev 2005; 27(3): 164-171. 